\title{
Search strategies to identify reports on "off-label" drug use in EMBASE
}

Bita Mesgarpour ${ }^{1,2}$, Markus Müller ${ }^{1}$ and Harald Herkner ${ }^{2^{*}}$

\begin{abstract}
Background: Medications are frequently prescribed outside their regulatory approval (off-label) by physicians particularly where appropriate therapies are not available. However, the risk/benefit ratio of drugs in off-label use needs to be critically appraised because it may differ from approved on-label usage. Therefore, an extensive exploration of current evidence on clinical data is well-advised. The objective of this study was to develop a search strategy that facilitates detection of the off-label drug use documents in EMBASE via OvidSP.

Methods: We constructed two sets of gold standards from relevant records to off-label drug use by a sensitive search of MEDLINE and EMBASE. Search queries, including search words and strings, were conceived based on definition of off-label use of medications as well as text analysis of 500 randomly selected relevant documents. The selected terms were searched in EMBASE (from 1988 to 2011) and their retrieval performance was compared with the gold standards. We developed a sensitivity-maximizing, and a sensitivity- and precision-maximizing search strategy.

Results: From 4067 records relevant to off-label drug use in our full gold standard set, 3846 records were retrievable from EMBASE. "off label*.af." was the most sensitive single term (overall sensitivity $77.5 \%$, sensitivity within EMBASE 81.9\%, precision 88.1\%). The highest sensitive search strategy was achieved by combining 36 search queries with overall sensitivity of $94.0 \%$ and precision of $69.5 \%$. An optimal sensitive and precise search strategy was yielded precision $87.4 \%$ at the expense of decreasing overall sensitivity to $89.4 \%$.
\end{abstract}

Conclusion: We developed highly sensitive search strategies to enhance the retrieval of studies on off-label drug use in OvidSP EMBASE.

Keywords: Off-label use, Information retrieval, EMBASE, MEDLINE, Sensitivity

\section{Background}

Pharmacotherapy is usually based on drugs that are approved for specific indications, dosages, routes of administration, or populations. However, administration of drugs outside these approved purposes is possible and denoted "off-label drug use". Off-label drug use is common practice, but generally suffers from a lack of sufficient evidence on risk/benefit assessment [1-4]. For some drugs off-label use is clinically more important than for approved purposes. For example, in a recent report off-label use of factor VII was found to be more frequent than its on-label indications [5]. Nonetheless,

\footnotetext{
* Correspondence: harald.herkner@meduniwien.ac.at

${ }^{2}$ Department of Emergency Medicine, Medical University Vienna, General Hospital, Währinger Gürtel 18-20, Vienna 1090, Austria

Full list of author information is available at the end of the article
}

there is increased effort to provide an evidence base for off-label use of drugs. Therefore, it appears important to explore the best strategy to find as many relevant studies as possible.

Free access to MEDLINE through the PubMed interface and its broad coverage makes it the first-choice database in biomedical literature [6,7]. Excerpta Medica Database (EMBASE) is also considered as a major bibliographic database in biomedicine but it is only available by subscription $[8,9]$. EMBASE covers pharmacology, pharmaceutical science and clinical research as its main areas of interest. In comparison with MEDLINE, it provides more extensive coverage of European and nonEnglish language publications [10] as well as conference abstracts [9].

\section{Biomed Central}

(c) 2012 Mesgarpour et al.; licensee BioMed Central Ltd. This is an Open Access article distributed under the terms of the Creative Commons Attribution License (http://creativecommons.org/licenses/by/2.0), which permits unrestricted use, distribution, and reproduction in any medium, provided the original work is properly cited. 
Accordingly, systematic searches restricted to MEDLINE only are generally not advised, because of potential introduction of retrieval bias [11-14]. Moreover, each bibliographic database has different indexing practice and thesaurus system. For example, records in MEDLINE are indexed using the National Library of Medicine's controlled vocabulary Medical Subject Headings $\left(\mathrm{MeSH}^{\circledR}\right)$ and EMBASE uses a thesaurus called EMTREE, which includes medical terms, drug names, acronyms, $\mathrm{MeSH}^{\circledR}$ headings and spelling variations. Therefore, even within one interface like OvidSP a search strategy is specific for a particular database. It is unclear whether a search strategy can be directly translated for applying in other databases, without loss of sensitivity or precision [15]. This may result in missing studies or retrieving many irrelevant documents. In a recent study, we found that MEDLINE did not cover $46 \%$ of off label drug use studies [16]. As a consequence we set out here to report the retrieval properties of selected search queries and combined queries for identifying offlabel drug use studies in EMBASE.

\section{Methods}

Our methods are detailed elsewhere [16]. Briefly, we did a systematic and sensitive search in MEDLINE and EMBASE through OvidSP (from 1948 and 1988, respectively; last updated in 28 February 2011) to find studies on off-label use of drugs. We constructed two sets of gold standards: the external or full set gold standard contained the whole relevant records retrieved from these two databases; the internal gold standard was the subset of documents indexed in EMBASE. Search queries and strategies were then created and tested for their
EMBASE), text words and strings by text analysis of 500 random documents in the gold standard full set. We applied frequently used fields in OvidSP EMBASE for searching like ".af." for all searchable fields, ".ab." for abstract, ".ti." for title and ".mp.", which restricts OvidSP's search to the text of title, abstract, subject headings, heading word, drug trade name, original title, device manufacturer or drug manufacturer name. We also used the Boolean operators "OR", "NOT", and proximity operator "ADJ[Number]", as well as truncation and wildcards.

We considered documents as relevant to off-label drug use, if they referred to any human drug outside the approval purposes, in terms of different dose, indication, route of application or for another age group. We did not apply any language restrictions, but method-wise we excluded book series, videos, errata, and corrections. We created a Microsoft (MS) Access database to store and manage all records retrieved by the search queries.

We compared the retrieval performance of each candidate term and combination of queries with the internal gold standard reference set by determining their sensitivity, precision and number needed to read (NNR). We chose an external gold standard in addition to the EMBASE gold standard to establish the general performance of our search strategies, supplementary to the performance which is driven by EMBASE indexing. Hence, we defined "overall sensitivity" as the number of relevant records in EMBASE retrieved by a search query/strategy divided by the total number of relevant records in the full gold standard set.

Sensitivity for a given search is defined as the proportion of relevant records retrieved from the database:

$$
\text { Sensitivity }=\frac{\text { number of relevant records in a database retrieved by a search strategy }}{\text { total number of relevant records in the database }} \times 100
$$

ability to retrieve relevant records in EMBASE. We assigned a search query as one line in a search strategy. It is usually a text string containing the exact sequence
Precision is the proportion of relevant records retrieved in the search, which is equivalent to positive predictive value:

$$
\text { Precision }=\frac{\text { number of relevant records retrieved by a search strategy }}{\text { total number of records retrieved by the search strategy }} \times 100
$$

of words and/or characters, and may include Boolean or proximity operators. Consequently, a search strategy consisted of several search queries connected with Boolean operators.

To construct a comprehensive set of possible search queries, we expanded our list of controlled vocabulary search terms or subject headings (EMTREE in
The NNR refers to the number of non-relevant records that one has to screen to find one of relevance [16]:

$$
\mathrm{NNR}=\frac{1}{\text { precision }} \times 100
$$


We ultimately aimed at developing a highly sensitive search strategy (HSSS) with two optimized versions: (1) a sensitivity-maximizing version and (2) a sensitivityand precision-maximizing version.

For the development of search strategies to optimize sensitivity or precision, we tested all combination of search queries by using an algorithm programmed in $\mathrm{C}+$ + . The search strategy with the highest balance of sensitivity and precision was developed by creating the scatter plot of precision versus sensitivity and calculating their best trade-off for the combined queries.

For hypothesis testing we used the Fisher's exact test for independent data and the McNemar test for correlated data with a two-sided p-value $<0.05$ to declare statistical significance.

\section{Results}

\section{Retrieved documents}

From 6,785 unique records, which were retrieved by a systematic search in MEDLINE and EMBASE via OvidSP, we classified 4,067 as relevant records to offlabel drug use. This constituted our full gold standard set (Figure 1). This set included 3,846 records retrievable within EMBASE, which was $62.5 \%$ of total retrieved records in EMBASE; among them, 3,480 (90.5\%) were published between years 2001-2011.

\section{Search results}

We evaluated the performance of 77 search queries and their combinations. In Table 1, we present the performance of the 15 search queries with the highest sensitivity (see Additional file 1 for the full list). "off label".af." yielded the highest sensitivity among the single terms (overall sensitivity $77.5 \%$, sensitivity within EMBASE $81.9 \%$, precision $88.1 \%$ ). Most of the top performing search queries had NNR of 1.1, which indicates that almost every abstract read classifies on off-label drug use. Truncation "off label" resulted in retrieving 35 more records, of which 31 (88.6\%) were relevant. It retrieved more relevant records than "(off adj2 label*).mp.", although the latter retrieved two unique records.

The overall sensitivity of search query "off label" and its truncation increased by $10.4 \%$ when we broadened their field from abstract (.ab.) to abstract and title (.ab,ti.). The most specific subject heading was "off label drug use". Exploding this to all its subheadings, "off label drug use".sh., resulted in $36.8 \%$ overall sensitivity (sensitivity within EMBASE 38.9\%). Truncating the text string "off label drug use" and then applying all field (.af.) slightly increased its overall sensitivity to $38.1 \%$ and $38.8 \%$, respectively.

We retrieved substantial non-relevant records after searching "label adj1 us*.af." consisted of the following terms: "using label-free" indicating different types of label-free proteomics technologies; "extra-label use"

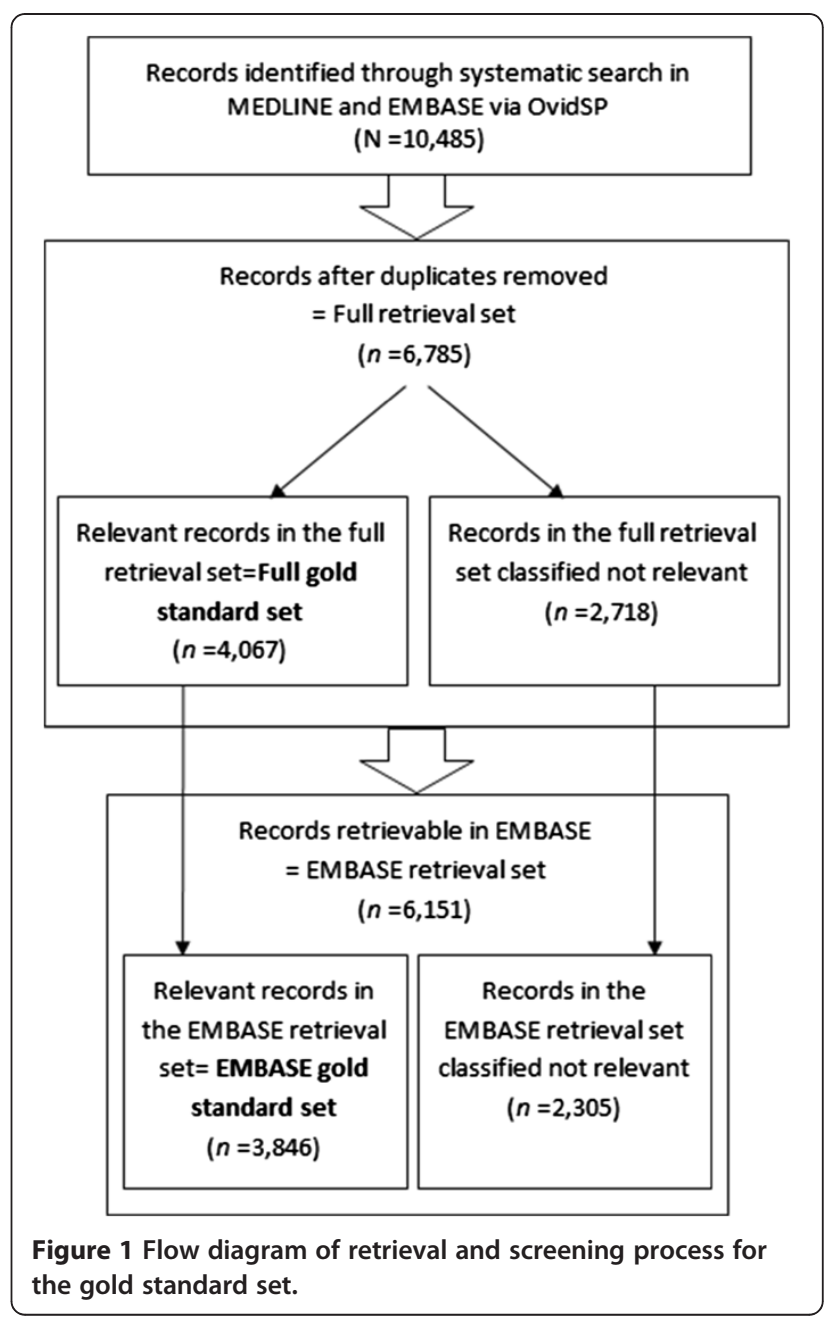

referring the off-label use of veterinary drugs; "openlabel use" as a type of study; "spin label using" specifying a tool to study the structure of proteins and biological membranes; and "food/nutritional label use" ascribing the information provided on food products.

To improve the precision of search term "inappropriate us $^{* *}$, we excluded the records about rational drug use and polypharmacy by NOTing out "antibiotic" or antimicrobial". The precision of search term "unlicense*. af." was improved by $30 \%$ and its NNR declined about two fold by eliminating retrieval of irrelevant content. It resulted by NOTing out an ORed string of 31 search terms prevalent in its irrelevant records retrieved (see Additional file 1).

To find the relevancy of some retrieved records, particularly those with no abstract such as the letters we had to read their full text. Whenever we detected some relevant editorial, letter or commentaries on other studies, we checked the retrieval status of cited study, consequently. In some cases, we found that we had not retrieved the relevant cited study through our search 
Table 1 Sensitivity, precision and number needed to read (NNR) of the 15 search queries in OvidSP EMBASE with the highest sensitivity (see the complete list in Additional file 1)

\begin{tabular}{|c|c|c|c|c|c|c|}
\hline Queries & $\begin{array}{l}\text { Number of relevant } \\
\text { records retrieved }\end{array}$ & $\begin{array}{c}\text { Number of records } \\
\text { retrieved }\end{array}$ & $\begin{array}{c}\text { Sensitivity/full } \\
\text { set (\%) }\end{array}$ & $\begin{array}{c}\text { Sensitivity/EMBASE } \\
\text { set (\%) }\end{array}$ & $\begin{array}{c}\text { Precision } \\
(\%)\end{array}$ & NNR \\
\hline off label*.af. & 3150 & 3577 & 77.5 & 81.9 & 88.1 & 1.1 \\
\hline (off adj2 label*).mp. & 3131 & 3589 & 77.0 & 81.4 & 87.2 & 1.1 \\
\hline (off adj1 label*).mp. & 3130 & 3567 & 77.0 & 81.4 & 87.7 & 1.1 \\
\hline off label*.mp. & 3128 & 3555 & 76.9 & 81.3 & 88.0 & 1.1 \\
\hline off label.af. & 3119 & 3542 & 76.7 & 81.1 & 88.1 & 1.1 \\
\hline (off adj1 label).mp. & 3119 & 3544 & 76.7 & 81.1 & 88.0 & 1.1 \\
\hline (off adj2 label).mp. & 3119 & 3547 & 76.7 & 81.1 & 87.9 & 1.1 \\
\hline off label.mp. & 3117 & 3540 & 76.6 & 81.0 & 88.1 & 1.1 \\
\hline "off label*".ab,ti. & 2306 & 2696 & 56.7 & 60.0 & 85.5 & 1.2 \\
\hline off label.ab,ti. & 2293 & 2679 & 56.4 & 59.6 & 85.6 & 1.2 \\
\hline "off label*".ab. & 1882 & 2224 & 46.3 & 48.9 & 84.6 & 1.2 \\
\hline off label.ab. & 1870 & 2208 & 46.0 & 48.6 & 84.7 & 1.2 \\
\hline (drug adj2 label adj2 us*).af. & 1587 & 1719 & 39.0 & 41.3 & 92.3 & 1.1 \\
\hline (drug adj1 label adj1 us*).af. & 1581 & 1710 & 38.9 & 41.1 & 92.5 & 1.1 \\
\hline "off label drug us*".af. & 1580 & 1677 & 38.8 & 41.1 & 94.2 & 1.1 \\
\hline
\end{tabular}

"ADJn is a positional operator to retrieve records that contain the terms (in any order) within a specified number ( $\mathrm{n}$ ) of words of each other. The adj1 operator finds two terms next to each other in any order. The adj2 operator finds terms in any order and with one word (or none) between them.

queries. For example, we retrieved an editorial record [17] on a study of Acharya and associates [18] about offlabel use of ranibizumab in uveitis. However, none of our search queries could retrieve the respective original article, despite our text analysis showed that the term "off-label" has been used four times in the full-text of Acharya paper [18].

\section{Final search strategies}

We constructed the best performing search strategy in terms of maximized sensitivity by "OR" combination of 32 search queries. This resulted in an overall sensitivity of $93.98 \%$ (99.38\% within EMBASE) and a precision of $69.48 \%$ (Table 2). Enhancement of sensitivity and precision after incrementally ORing new search queries is shown as cumulative sensitivity and precision in Table 2 .

Overall sensitivity and precision of the EMBASE HSSS (sensitivity maximized) was higher in the recent years (2001-2011) compared to years 1988-2000 (95.6\% vs $79.3 \%$ and $75.0 \%$ vs $40.8 \%, \mathrm{p}<0.001$ for both), while sensitivity within EMBASE remained approximately constant (99.5\% vs 99.4\%; $\mathrm{p}=0.99$, Fisher exact test).

We also tested the EMBASE HSSS (sensitivity maximized) in the full gold standard set excluding 500 records that used to develop our strategies and received virtually the same results.

To evaluate the retrieval function of off-label drug use search strategy developed for a different database, we further tested our published search strategy for OvidSP MEDLINE [16] in OvidSP EMBASE. The retrieval implications of re-running our published highly sensitive search strategy A for OvidSP MEDLINE (MEDLINE HSSS) in EMBASE yielded an overall sensitivity of $89 \%$ and a sensitivity within EMBASE of 94\%, precision of this search strategy was $77.7 \%$. Using the EMBASE HSSS (sensitivity maximized) compared with the published MEDLINE HSSS (sensitivity maximized) in EMBASE increased sensitivity from $94 \%$ to $99 \%$ ( $p<0.001$, McNemar test). Out of 4,067 studies classified relevant in the full set gold standard, 1,942 studies were retrieved through EMBASE HSSS in EMBASE as well as the MEDLINE HSSS in MEDLINE (both sensitivity maximized). By searching in MEDLINE only, 1,880 relevant records were not retrieved whereas by searching in EMBASE only, 222 were not retrieved ( $\mathrm{p}<0.001$, McNemar test).

We plotted precision versus overall sensitivity of search query combinations to optimize sensitivity and precision (Figure 2). Table 3 shows the most parsimonious search strategy amongst 15 strategies where sensitivity was optimized for high precision (see Additional file 2). The combination of 22 search queries and query "(stent" or veterinar*).af." by using a NOT operator eliminated the irrelevant records out of the most optimized sensitive and precise search strategy leading to $5.32 \%$ improvement of precision at the cost of a small decrease in sensitivity (overall and within EMBASE 0.37\% and 0.39\%, respectively).

\section{OvidSP EMBASE characteristics}

A substantial change in number of retrieved records in EMBASE was found in early August 2010. 206 duplicated and two triplicated records were retrieved in our 
Table 2 Highly Sensitive Search Strategy for identifying off-label drug use reports in OvidSP EMBASE: sensitivitymaximizing version

\begin{tabular}{|c|c|c|c|c|c|c|}
\hline & Search query & $\begin{array}{c}\text { Cumulative number } \\
\text { of relevant records } \\
\text { retrieved }\end{array}$ & $\begin{array}{c}\text { Cumulative } \\
\text { number of records } \\
\text { retrieved }\end{array}$ & $\begin{array}{c}\text { Cumulative } \\
\text { sensitivity/full } \\
\text { set (\%) }\end{array}$ & $\begin{array}{c}\text { Cumulative } \\
\text { sensitivity/EMBASE } \\
\text { set (\%) }\end{array}$ & $\begin{array}{l}\text { Cumulative } \\
\text { Precision (\%) }\end{array}$ \\
\hline 1 & off label*.af. & 3150 & 3577 & 77.45 & 81.90 & 88.06 \\
\hline 2 & (off adj1 label).mp. & 3152 & 3581 & 77.50 & 81.96 & 88.02 \\
\hline 3 & (drug adj2 label adj2 us*).af. & 3153 & 3617 & 77.53 & 81.98 & 87.17 \\
\hline 4 & unlicense*.af. & 3279 & 4320 & 80.62 & 85.26 & 75.90 \\
\hline 5 & unapprove*.af. & 3445 & 4635 & 84.71 & 89.57 & 74.33 \\
\hline 6 & (label adj3 indication*).af. & 3450 & 4667 & 84.83 & 89.70 & 73.92 \\
\hline 7 & off li?en? e*.af. & 3509 & 4742 & 86.28 & 91.24 & 74.00 \\
\hline 8 & $\begin{array}{l}\text { ((no* licen?ed for adj3 use* }) \\
\text { not now licen?ed).af. }\end{array}$ & 3560 & 4825 & 87.53 & 92.56 & 73.78 \\
\hline$\overline{9}$ & $\begin{array}{l}\text { ((inappropriate } \text { us* }^{*} \text { and indication) } \\
\text { not (antibiotic } \text { or antimicrobial)).af. }\end{array}$ & 3609 & 4964 & 88.74 & 93.84 & 72.70 \\
\hline 10 & $\begin{array}{l}\text { ((appropriate* adj3 prescri*) and } \\
\text { indication).af. }\end{array}$ & 3648 & 5081 & 89.70 & 94.85 & 71.80 \\
\hline 11 & (outside adj3 licen?e*).af. & 3665 & 5110 & 90.12 & 95.29 & 71.72 \\
\hline 12 & unlabel* us*.af. $^{*}$ & 3692 & 5140 & 90.78 & 96.00 & 71.83 \\
\hline 13 & labeled indication*.af. & 3703 & 5169 & 91.05 & 96.28 & 71.64 \\
\hline$\overline{14}$ & (inappropriate indication*).af. & 3719 & 5294 & 91.44 & 96.70 & 70.25 \\
\hline 15 & nonapprove*.af. & 3737 & 5337 & 91.89 & 97.17 & 70.02 \\
\hline 16 & registered indication*.af. & 3752 & 5367 & 92.25 & 97.56 & 69.91 \\
\hline 17 & offlabel*.af. & 3757 & 5372 & 92.38 & 97.69 & 69.94 \\
\hline 18 & (out* adj4 licen?ed indication*).af. & 3759 & 5376 & 92.43 & 97.74 & 69.92 \\
\hline 19 & (unlabel* adj3 indication*).af. & 3767 & 5384 & 92.62 & 97.95 & 69.97 \\
\hline 20 & non fda approve*.af. & 3780 & 5411 & 92.94 & 98.28 & 69.86 \\
\hline 21 & $\begin{array}{l}\text { ((no* licen?ed for adj3 indication*) } \\
\text { not now licen?ed).af. }\end{array}$ & 3793 & 5425 & 93.26 & 98.62 & 69.92 \\
\hline 22 & (appropriate indication adj3 us*).af. & 3796 & 5432 & 93.34 & 98.70 & 69.88 \\
\hline 23 & (be???d* adj2 licen?ed indication*).af. & 3799 & 5436 & 93.41 & 98.78 & 69.89 \\
\hline 24 & (us* without adj2 indication*).af. & 3804 & 5445 & 93.53 & 98.91 & 69.86 \\
\hline 25 & (prescri* outside adj4 guideline*).af. & 3808 & 5450 & 93.63 & 99.01 & 69.87 \\
\hline 26 & (out of label).af. & 3810 & 5466 & 93.68 & 99.06 & 69.70 \\
\hline 27 & (improper adj1 indication*).af. & 3812 & 5472 & 93.73 & 99.12 & 69.66 \\
\hline 28 & $\begin{array}{l}\text { (inappropriate adj5 indication adj2 } \\
\text { us*).af. }\end{array}$ & 3814 & 5475 & 93.78 & 99.17 & 69.66 \\
\hline 29 & no* appropriate indication*.af. & 3815 & 5482 & 93.80 & 99.19 & 69.59 \\
\hline 30 & (non evidence base* us*).af. $^{*}$ & 3818 & 5487 & 93.88 & 99.27 & 69.58 \\
\hline 31 & without proper indication*.af. & 3821 & 5498 & 93.95 & 99.35 & 69.50 \\
\hline 32 & $\begin{array}{l}\text { (or/1-31) or (drug* without adj2 } \\
\text { indication*).af. }\end{array}$ & 3822 & 5501 & 93.98 & 99.38 & 69.48 \\
\hline
\end{tabular}

Combinations of search queries with the best sensitivity are ranked in descending order of number of relevant records.

last search update. They were indexed in two different accession numbers but mostly in the same entry week. For example, a study by Caron [19] had two records with two accession numbers and similar entry week (17872714 and 2007205136; entry week: 200700) and a study by Daskalaki et al. [20] had two records with two accession numbers and two entry weeks: 18595974 (entry week: 200800) and 2009161626 (entry week:
200900) (see Additional file 3). Moreover, we came across to some other errors such as dup/triplicate records because of typographical errors or more than one translation for non-English titles. For example, a study by Konda et al. [21] recorded in two different titles "Colchicine in dermatology" and "Dosages and administration". The latter title is wrong and this record has also failed to provide author's name and had a typo error in 


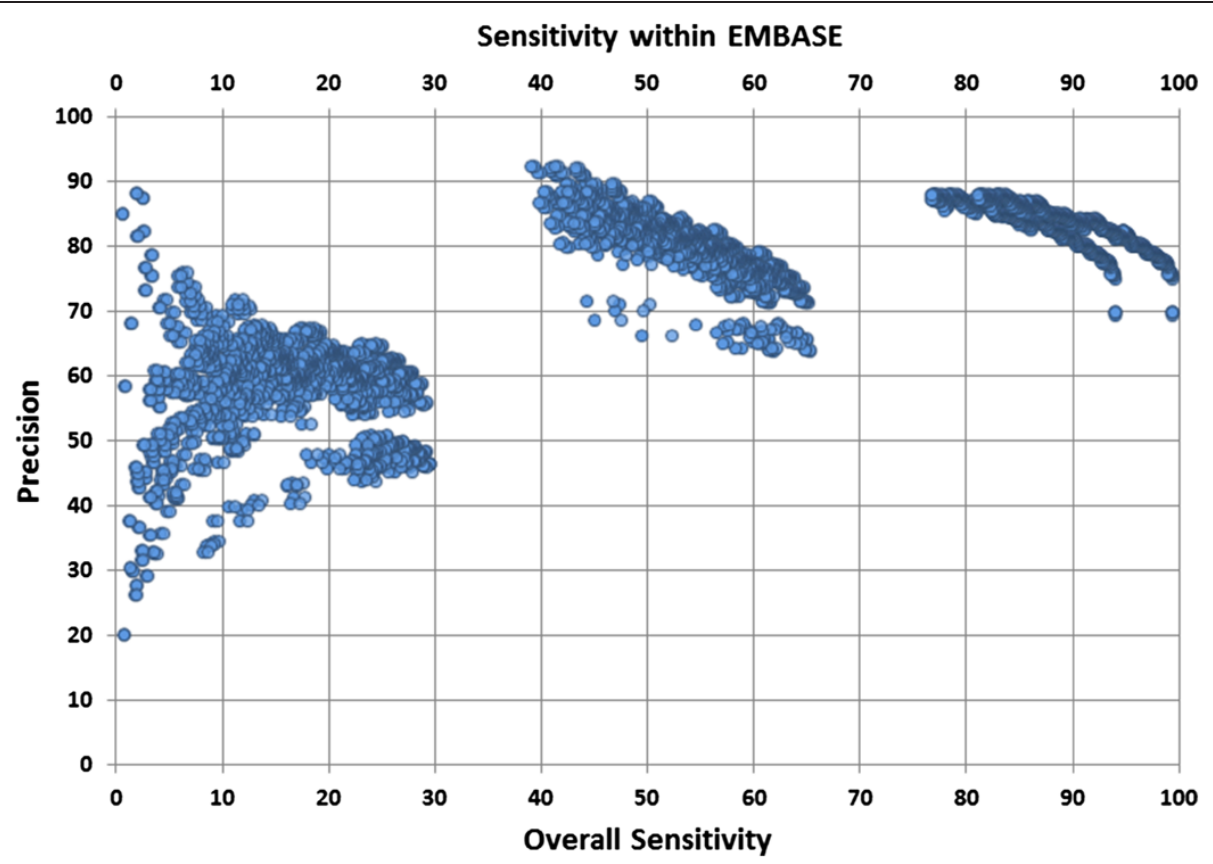

Figure 2 Plot of sensitivities (relevant documents in the overall set $(n=4,067)$ and within EMBASE $(n=3,846))$ versus precision for different combinations of search queries to detect studies on off-label drug usage in electronic databases.

start page (202 instead of 201). The correct title was indexed with 10 subject headings and the wrong one with 56 subject headings including "off label drug use" (see Additional file 3). We also found that indexing for documents that are published in more than one journal might be different. It is also the case when a document is published as both a conference abstract and an article. For example, a study by De Jong et al. which is published as a conference abstract [22] and an article [23] was indexed with 15 subject headings where publication type was 'abstract' and with 36 subject headings including "off label drug use" where publication type was 'article'. The abstracts recorded for these two publication types were almost the same (see Additional file 3). However, the database issues presented do not appear to explain much of the performance limitations overall.

The sensitivity and precision of some search queries was different after replacing the "af" field by "mp" in our last update, 28 February 2011. Therefore, we chose the best balance of sensitivity and precision (see Additional file 1). However, running our search queries in 30 January 2012 showed that the discrepancies between these two search fields have been removed. Updating issues during the integration of records from MEDLINE into the OvidSP EMBASE database may explain this (e-mail communication with Ovid Training Department).

\section{Discussion}

We developed highly sensitive search strategies for OvidSP EMBASE to enhance the retrieval of studies on off-label drug use. These highly sensitive search strategies outperformed single search terms, EMTREE terms, and queries as well as search strategies designed for another database.

Top-performing queries were almost the same in EMBASE compared to our published search queries developed for MEDLINE [16]. For example, the queries "off label*.af.", "(off adj2 label ${ }^{*}$ ).mp." and "(off adj1 label").mp." were all top performers in both databases. Three queries "(unlabel" adj3 indication*).af.", "(unapprove* adj5 prescription).af." and "(outside adj3 licen? $\mathrm{e}^{*}$ ). af." were only selected for EMBASE. We also chose "off label.mp." because of different retrieval for search fields "af" and "mp" in EMBASE. Furthermore, the most specific subject heading was not exactly identical in two databases: "off label drug use" in EMBASE and "OffLabel Use" in MEDLINE.

Optimized strategies, sensitivity-maximizing version and sensitivity- and precision-maximizing version, in EMBASE had greater overall sensitivity (sensitivity in the full gold standard set) and precision than the comparable MEDLINE strategy. However, the sensitivity within their internal gold standard was almost the same. This can be best explained by different coverage of these databases. For example, a study showed that $47 \%$ of 386 Syrian Arab Republic reports indexed in MEDLINE or EMBASE were found exclusively in EMBASE, while 32\% from MEDLINE alone and 21\% from both of them [24]. Moreover, by the integration of records from MEDLINE into EMBASE, the coverage of EMBASE must have increased [25]. 
Table 3 Highly sensitive search strategy for identifying off-label drug use reports in OvidSP EMBASE: sensitivity- and precision-maximizing version

\begin{tabular}{|c|c|c|c|c|c|c|}
\hline & Search query & $\begin{array}{l}\text { Cumulative } \\
\text { number of } \\
\text { relevant records } \\
\text { retrieved }\end{array}$ & $\begin{array}{c}\text { Cumulative } \\
\text { number of } \\
\text { records } \\
\text { retrieved }\end{array}$ & $\begin{array}{c}\text { Cumulative } \\
\text { sensitivity/full } \\
\text { set }(\%)\end{array}$ & $\begin{array}{c}\text { Cumulative } \\
\text { sensitivity/EMBASE } \\
\text { set (\%) }\end{array}$ & $\begin{array}{l}\text { Cumulative } \\
\text { Precision } \\
\text { (\%) }\end{array}$ \\
\hline 1 & off label*.af. & 3150 & 3577 & 77.45 & 81.90 & 88.06 \\
\hline 2 & $\begin{array}{l}\text { (unlicensed not (unlicensed aide* or unlicensed assist* or } \\
\text { unlicensed car* or (Unlicensed adj2 heal*)or unlicensed } \\
\text { home }^{*} \text { or (killer adj2 cell*) or (unlicensed adj2 individual*) } \\
\text { or (unlicensed adj2 nurs*) or (unlicensed adj4 practi*) or } \\
\text { (unlicensed adj2 physician*) or (unlicensed adj2 operat*) } \\
\text { or (unlicensed adj2 person*) or unlicensed profession* or } \\
\text { unlicensed rid* or (unlicensed adj3 staff*) or unlicensed } \\
\text { therapist* or (unlicensed adj5 vaccine*) or unlicensed } \\
\text { vendor* or (unlicensed adj2 work*) or (unlicensed adj2 } \\
\text { employe* or device*or dentist*or driver or driving or } \\
\text { herbal or medical graduate* or motor* or pesticide*or } \\
\text { premis*or prostitute } \text { or }^{*} \text { restaurant*or veterinary or } \\
\text { worker*)).af., }\end{array}$ & 3272 & 3858 & 80.45 & 85.08 & 84.81 \\
\hline 3 & (unapprove* adj2 us*).af. & 3331 & 3947 & 81.90 & 86.61 & 84.39 \\
\hline 4 & (unapprove* adj5 indication*).af. & 3367 & 3986 & 82.79 & 87.55 & 84.47 \\
\hline 5 & off li?en?e.af. & 3425 & 4053 & 84.21 & 89.05 & 84.51 \\
\hline 6 & ((no* licen?ed for adj3 use*) not now licen?ed).af. & 3476 & 4136 & 85.47 & 90.38 & 84.04 \\
\hline 7 & (unapprove* adj2 drug*).af. & 3493 & 4181 & 85.89 & 90.82 & 83.54 \\
\hline 8 & (outside adj3 licen?e*).af. & 3511 & 4211 & 86.33 & 91.29 & 83.38 \\
\hline 9 & unlabel* us*.af. & 3538 & 4241 & 86.99 & 91.99 & 83.42 \\
\hline 10 & labeled indication*.af. & 3549 & 4270 & 87.26 & 92.28 & 83.11 \\
\hline 11 & nonapprove*.af. & 3569 & 4317 & 87.76 & 92.80 & 82.67 \\
\hline 12 & registered indication*.af. & 3585 & 4348 & 88.15 & 93.21 & 82.45 \\
\hline 13 & offlabel*.af. & 3590 & 4353 & 88.27 & 93.34 & 82.47 \\
\hline 14 & (unlabel* adj3 indication*).af. & 3598 & 4361 & 88.47 & 93.55 & 82.50 \\
\hline 15 & non fda approve*.af. & 3611 & 4388 & 88.79 & 93.89 & 82.29 \\
\hline 16 & ((no* licen?ed for adj3 indication*) not now licen?ed).af. & 3624 & 4402 & 89.11 & 94.23 & 82.33 \\
\hline 17 & (appropriate indication adj3 us*).af. & 3630 & 4413 & 89.25 & 94.38 & 82.26 \\
\hline 18 & (be???d* adj2 licen?ed indication*).af. & 3633 & 4417 & 89.33 & 94.46 & 82.25 \\
\hline 19 & (us* without adj2 indication*).af. & 3638 & 4428 & 89.45 & 94.59 & 82.16 \\
\hline 20 & (prescri* outside adj4 guideline*).af. & 3643 & 4434 & 89.57 & 94.72 & 82.16 \\
\hline 21 & (inappropriate adj5 indication adj2 us*).af. & 3647 & 4441 & 89.67 & 94.83 & 82.12 \\
\hline 22 & (non evidence base* us*).af. $^{*}$ & 3650 & 4446 & 89.75 & 94.90 & 82.10 \\
\hline 23 & (or/1-22) not (stent* or veterinar*).af. & 3635 & 4158 & 89.38 & 94.51 & 87.42 \\
\hline
\end{tabular}

Combinations of search queries with the best optimization of sensitivity and precision are ranked in descending order of number of relevant records. See further search strategies in Additional file 2.

The necessity for developing a specific search strategy adapted to the indexing structure, limits, and special features for each database is extensively reported, although there is no study on retrieval failure [15]. Re-running a highly sensitive search strategy which we developed for identifying off-label drug use in OvidSP MEDLINE [16] in OvidSP EMBASE achieved about 5\% less sensitivity compared to our newly developed search strategy, but $8.2 \%$ higher precision.

Retrieving relevant studies on off-label drug use might be influenced by many mechanisms such as low quality of off-label use reporting and poor indexing in bibliographic databases. Administration of medication for unapproved purposes is not well defined or properly described in some reports. This might happen when authors are inconsiderate or unfamiliar with the concept. A survey of 95 officebased pediatricians in France showed that they did not recognize the off-label status of 686 out of 745 (92\%) drug courses they commonly prescribed [26]. Another study in Northern Ireland on experience and attitudes of healthcare professionals on unlicensed/off-label pediatric prescribing revealed that $41 \%$ of 563 respondents were not familiar with the term "off-label medicines" prior to participating in the study [27]. 
Inadequate relevant keywords (as provided by study authors), inconsistent terminology or ambiguous description in the abstract might lead to the poor indexing, because the whole information contained in the full-text record is not detected by searching in bibliographic databases. Thus, low sensitivity of the specific EMTREE term for off-label drug use studies in EMBASE, "off label drug use".sh., confirmed inconsistent indexing. Another indication of poor indexing is the fact that in some cases secondary papers like letters, commentaries or editorials could be retrieved but not the original papers. Glanville, et al. showed that MEDLINE and EMBASE indexing terms for economic evaluations are not efficient either. They indicated that it could be explained by indexer uncertainty and indexing lapses, in addition to poor reporting by authors [28].

As a result of the low quality of reporting off-label drug use and poor indexing, we will not be able to retrieve all relevant records on off-label drug use by search in bibliographic databases alone, even with the highest sensitive search strategies. Thus, we suggest the implementation of our search strategy already for broad sweep research questions. "Off-label drug use in multiple sclerosis" or "off-label use of beta interferon" might serve as an example.

Search queries included in EMBASE HSSS were the same as MEDLINE HSSS in $81.3 \%$ (both sensitivity maximized strategy); the latter had one query less and the differences between three out of five dissimilar queries were due to the distance between two terms by using proximity operator. For example, "unlabel" indication". af." in MEDLINE HSSS was replaced by "(unlabel* adj3 indication*).af." in EMBASE HSSS. Among 23 queries included in EMBASE HSSS (sensitivity- and precisionmaximized), 11 queries were identical to the respective MEDLINE strategy. It also included a very long query (NOTed out "unlicensed") and two more queries than MEDLINE.

We found that using an exclusion strategy was efficient to develop the optimized sensitive and precise strategy. It resulted from attempts to remove irrelevant studies whilst retaining sensitivity in two phases. The first one resulted from improving the retrieval by eliminating irrelevant content with search term "unlicensed". In the second phase, we excluded studies on off-label use of medical devices, in particular stents, and veterinary medications from the selected set of queries, as it was the case in our prior study on developing a MEDLINE strategy [16]. It is likely that NOT operators impair sensitivity of a search $[29,30]$ due to removing relevant as well as irrelevant records. Alternatively, there is a rather safe approach to use this operator. For example, the Cochrane HSSS (sensitivity maximizing) for identifying randomized trials in MEDLINE consisted of a query "animals [mh] NOT humans [mh]" which is combined by NOT to OR string of eight search terms [31]. Thus, this strategy excludes reports solely of animal studies, but retains reports indexed as human and animal, and neither human nor animal. Hence, we chose the search terms to combine with NOT if they were frequently used in the irrelevant records and rarely appeared in the relevant records only. Nonetheless, the sensitivity was slightly reduced as a consequence of excluding some relevant records. A recent study showed that NOTing out irrelevant content could improve retrieval of original studies on diagnosis, prognosis and etiology in MEDLINE, EMBASE, CINAHL and PsycINFO [32]. We also truncated short words like "use" or "out" within an exact sequence of words only. The truncated short terms are not generally recommended because they could retrieve many different words yielded low precision due to retrieving irrelevant records and in some database interfaces unexpected results may happen. Therefore, we carefully followed these short terms with an "exact" sequence of words to decrease its possible "side effects" associated with irrelevant records retrieval.

\section{Conclusion}

We developed highly sensitive search strategies for OvidSP EMBASE to enhance the retrieval of studies on off-label drug use. Our study demonstrates that a comprehensive search for off-label drug use in OvidSP EMBASE can be much improved by highly sensitive search strategies instead of using simple search terms.

\section{Additional files}

Additional file 1: Table S1. Sensitivity, precision and number needed to read (NNR) of search queries in OvidSP EMBASE.

Additional file 2: Table S2. Combinations of search queries with the best optimization of sensitivity and precision for detecting off-label drug use reports in OvidSP EMBASE.

Additional file 3: OvidSP EMBASE Characteristics: Examples.

\section{Abbreviations}

Ab: Abstract; ab,ti: Abstract or title; af: All searchable fields; EMBASE: Excerpta Medica Database; HSSS: Highly sensitive search strategy; mh: Medical Subject Heading (MeSH) term ('exploded'); mp: Title, abstract, subject headings, heading word, drug trade name, original title, device manufacturer, drug manufacturer name; NNR: Number needed to read; sh: Medical Subject Headings; ti: Title.

\section{Competing interests}

The authors declare that they have no competing interests.

\section{Authors' contributions}

$\mathrm{HH}$ had the idea. All authors contributed to the design of the study and construction of the study protocol. BM researched data and discussed the results with all authors. $\mathrm{HH}$ was responsible for statistical evaluations and interpretation of data. BM wrote the first drafts of the paper, which were critically revised by $\mathrm{HH}$ and $\mathrm{MM}$. All authors read and approved the final manuscript. 


\section{Acknowledgements}

We would like to express our gratitude to Mohsen Mesgarpour for assisting us in analysis of the search queries data by developing a computer program.

\section{Author details}

'Department of Clinical Pharmacology, Medical University Vienna, General Hospital, Währinger Gürtel 18-20, Vienna 1090, Austria. ${ }^{2}$ Department of Emergency Medicine, Medical University Vienna, General Hospital, Währinger Gürtel 18-20, Vienna 1090, Austria.

Received: 5 July 2012 Accepted: 19 December 2012

Published: 29 December 2012

\section{References}

1. Alexander GC, Gallagher SA, Mascola A, Moloney RM, Stafford RS: Increasing off-label use of antipsychotic medications in the United States, 19952008. Pharmacoepidemiol Drug Saf 2011, 20:177-184.

2. de Souza JA: Advances in drug development: off-label drug utilization in oncology. Clin Adv Hematol Oncol 2011, 9:473-475.

3. Lat I, Micek S, Janzen J, Cohen H, Olsen K, Haas C: Off-label medication use in adult critical care patients. J Crit Care 2011, 26:89-94.

4. Lee E, Teschemaker AR, Johann-Liang R, Bazemore G, Yoon M, Shim KS, Daniel M, Pittman J, Wutoh AK: Off-label prescribing patterns of antidepressants in children and adolescents. Pharmacoepidemiol Drug Saf 2012, 21:137-144.

5. Logan AC, Yank V, Stafford RS: Off-label use of recombinant factor VIla in U.S. hospitals: analysis of hospital records. Ann Intern Med 2011, 154:516-522.

6. Ajuwon GA: Use of the Internet for health information by physicians for patient care in a teaching hospital in Ibadan, Nigeria. Biomed Digit Libr 2006, 3:12.

7. Sahapong S, Manmart L, Ayuvat D, Potisat S: Information use behavior of clinicians in evidence-based medicine process in Thailand. $J$ Med Assoc Thai 2009, 92:435-441.

8. Briggs K, Crowlesmith I: EMBASE-The excerpta medica database: Quick and comprehensive drug information. Publish Res Q 1995, 11:51-60.

9. University of York, NHS centre for reviews and dissemination: Finding studies for systematic reviews: a resource list for researchers. York: Centre for Reviews and Dissemination, University of York; 2010.

10. Wong SS, Wilczynski NL, Haynes RB: Comparison of top-performing search strategies for detecting clinically sound treatment studies and systematic reviews in MEDLINE and EMBASE. J Med Libr Assoc 2006, 94:451-455

11. Bahaadinbeigy $K$, Yogesan $K$, Wootton R: MEDLINE versus EMBASE and CINAHL for telemedicine searches. Telemed J E Health 2010, 16:916-919.

12. Kelly L, St Pierre-Hansen N: So many databases, such little clarity: Searching the literature for the topic aboriginal. Can Fam Physician 2008, 54:1572-1573.

13. Sampson M, Barrowman NJ, Moher D, Klassen TP, Pham B, Platt R, St John $P D$, Viola R, Raina P: Should meta-analysts search Embase in addition to Medline? J Clin Epidemio/ 2003, 56:943-955.

14. Wilkins T, Gillies RA, Davies K: EMBASE versus MEDLINE for family medicine searches: can MEDLINE searches find the forest or a tree? Can Fam Physician 2005, 51:848-849.

15. Sampson M, McGowan J, Lefebvre C, Moher D, Grimshaw J: PRESS: peer review of electronic search strategies. Ottawa: Canadian Agency for Drugs and Technologies in Health; 2008

16. Mesgarpour B, Müller M, Herkner H: Search strategies- identified reports on "off-label" drug use in MEDLINE. J Clin Epidemiol 2012, 65:827-834.

17. Gaudio PA: Ranibizumab for uveitic macular edema: why? Am J Ophthalmol 2009, 148:179-180.

18. Acharya NR, Hong KC, Lee SM: Ranibizumab for refractory uveitis-related macular edema. Am J Ophthalmol 2009, 148:303-309. e302.

19. Caron C: Practice tips. Inserting the levonorgestrel intrauterine system: off-label use. Can Fam Physician 2007, 53:643-644.

20. Daskalaki I, Spain CV, Long SS, Watson B: Implementation of rotavirus immunization in Philadelphia, Pennsylvania: high levels of vaccine ineligibility and off-label use. Pediatrics 2008, 122:e33-e38.

21. Konda C, Rao AG: Colchicine in dermatology. Indian J Dermatol Venereol Leprol 2010, 76:201-205.
22. de Jong J, van den Berg PB, Visser ST, de Vries TW, de Jong-van den Berg $\mathrm{LT}$ : Antibiotic usage, dosage and course length in children between 0 and 4 years. Pharmacoepidemiol Drug Saf 2009, 18:S213-S214.

23. de Jong J, van den Berg PB, Visser ST, de Vries TW, de Jong-van den Berg LT: Antibiotic usage, dosage and course length in children between 0 and 4 years. Acta Paediatr 2009, 98:1142-1148.

24. Matar HE, Almerie MQ, Adams CE, Essali A: Publications indexed in Medline and Embase originating from the Syrian Arab Republic: a survey. East Mediterr Health J 2009, 15:648-652.

25. Excerpta Medica Database (EMBASE): Release fact sheet (summer 2009). The Netherlands: Elsevier B.V; 2009. Available from http://www.embase.com/ info/UserFiles/Aug1ReleaseFactSheet_0.pdf.

26. Chalumeau M, Treluyer JM, Salanave B, Assathiany R, Cheron G, Crocheton N, Rougeron C, Mares M, Breart G, Pons G: Off label and unlicensed drug use among French office based paediatricians. Arch Dis Child 2000, 83:502-505

27. Mukattash T, Hawwa AF, Trew K, McElnay JC: Healthcare professional experiences and attitudes on unlicensed/off-label paediatric prescribing and paediatric clinical trials. Eur J Clin Pharmacol 2011, 67:449-461.

28. Glanville J, Kaunelis D, Mensinkai S: How well do search filters perform in identifying economic evaluations in MEDLINE and EMBASE. Int J Techno Assess Health Care 2009, 25:522-529.

29. Deacon P, Smith JB, Tow S: Using metadata to create navigation paths in the HealthInsite Internet gateway. Health Info Libr J 2001, 18:20-29.

30. Jenuwine ES, Floyd JA: Comparison of medical subject headings and textword searches in MEDLINE to retrieve studies on sleep in healthy individuals. J Med Libr Assoc 2004, 92:349-353.

31. Lefebvre C, Manheimer E, Glanville J: Chapter 6: Searching for studies. In Cochrane handbook for systematic reviews of interventions version 5.1.0 (Updated march 2011). Edited by Higgins JPT, Green S, The Cochrane Collaboration; 2011. Available from www.cochrane-handbook.org.

32. Wilczynski NL, McKibbon KA, Haynes RB: Search filter precision can be improved by NOTing out irrelevant content. AMIA Annu Symp Proc 2011 2011:1506-1513.

doi:10.1186/1471-2288-12-190

Cite this article as: Mesgarpour et al:: Search strategies to identify reports on "off-label" drug use in EMBASE. BMC Medical Research Methodology 2012 12:190.

\section{Submit your next manuscript to BioMed Central and take full advantage of:}

- Convenient online submission

- Thorough peer review

- No space constraints or color figure charges

- Immediate publication on acceptance

- Inclusion in PubMed, CAS, Scopus and Google Scholar

- Research which is freely available for redistribution 